\title{
Detection of the Geminga pulsar with MAGIC hints at a power-law tail emission beyond $15 \mathrm{GeV}$
}

MAGIC Collaboration: V. A. Acciari ${ }^{1,2}$, S. Ansoldi ${ }^{3}$, L. A. Antonelli ${ }^{4}$, A. Arbet Engels ${ }^{5}$, K. Asano ${ }^{6}$, D. Baack ${ }^{7}$, A. Babic ${ }^{8}$, A. Baquero ${ }^{9}$, U. Barres de Almeida ${ }^{10}$, J. A. Barrio ${ }^{9}$, J. Becerra González ${ }^{1,2}$, W. Bednarek ${ }^{11}$, L. Bellizzi ${ }^{12}$, E. Bernardini ${ }^{13}$, M. Bernardos ${ }^{14}$, A. Berti ${ }^{15}$, J. Besenrieder ${ }^{16}$, W. Bhattacharyya ${ }^{13}$, C. Bigongiari ${ }^{4}$, A. Biland ${ }^{5}$, O. Blanch ${ }^{17}$, G. Bonnoli ${ }^{12}$, Ž. Bošnjak ${ }^{8}$, G. Busetto ${ }^{18}$, R. Carosi ${ }^{17}$, G. Ceribella ${ }^{16, \star}$, M. Cerruti ${ }^{20}$, Y. Chai ${ }^{16}$, A. Chilingarian ${ }^{21}$, S. Cikota ${ }^{8}$, S. M. Colak ${ }^{17}$, E. Colombo ${ }^{1,2}$, J. L. Contreras ${ }^{9}$, J. Cortina ${ }^{14}$, S. Covino ${ }^{4}$, G. D’Amico ${ }^{16}$, V. D’Elia ${ }^{4}$, P. Da Vela ${ }^{19,36}$, F. Dazzi ${ }^{4}$, A. De Angelis ${ }^{18}$, B. De Lotto ${ }^{3}$, M. Delfino ${ }^{17,37}$, J. Delgado ${ }^{17,37}$, C. Delgado Mendez ${ }^{14}$, D. Depaoli ${ }^{15}$, T. Di Girolamo ${ }^{15}$, F. Di Pierro ${ }^{15}$, L. Di Venere ${ }^{15}$, E. Do Souto Espiñeira ${ }^{17}$, D. Dominis Prester $^{22}$, A. Donini ${ }^{3}$, D. Dorner ${ }^{23}$, M. Doro ${ }^{18}$, D. Elsaesser ${ }^{7}$, V. Fallah Ramazani ${ }^{24}$, A. Fattorini ${ }^{7}$, G. Ferrara ${ }^{4}$, L. Foffano ${ }^{18}$, M. V. Fonseca ${ }^{9}$, L. Font ${ }^{25}$, C. Fruck ${ }^{16}$, S. Fukami ${ }^{6}$, R. J. García López ${ }^{1,2}$,

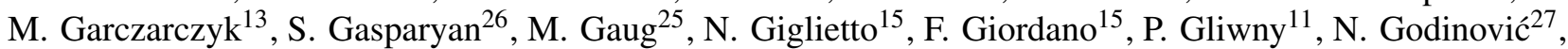
J. G. Green ${ }^{4}$, D. Green ${ }^{16}$, D. Hadasch ${ }^{6}$, A. Hahn ${ }^{16}$, L. Heckmann ${ }^{16}$, J. Herrera ${ }^{1,2}$, J. Hoang ${ }^{9}$, D. Hrupec ${ }^{28}$,

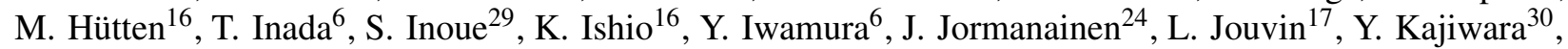
M. Karjalainen ${ }^{1,2}$, D. Kerszberg ${ }^{17}$, Y. Kobayashi ${ }^{6}$, H. Kubo ${ }^{30}$, J. Kushida ${ }^{31}$, A. Lamastra ${ }^{4}$, D. Lelas ${ }^{27}$, F. Leone ${ }^{4}$, E. Lindfors ${ }^{24}$, S. Lombardi ${ }^{4}$, F. Longo ${ }^{3,38}$, R. López-Coto ${ }^{18}$, M. López-Moya ${ }^{9, \star}$, A. López-Oramas ${ }^{1,2}$,

S. Loporchio ${ }^{15}$, B. Machado de Oliveira Fraga ${ }^{10}$, C. Maggio ${ }^{25}$, P. Majumdar ${ }^{32}$, M. Makariev ${ }^{33}$, M. Mallamaci ${ }^{18}$, G. Maneva ${ }^{33}$, M. Manganaro ${ }^{22}$, K. Mannheim ${ }^{23}$, L. Maraschi ${ }^{4}$, M. Mariotti ${ }^{18}$, M. Martínez $^{17}$, D. Mazin ${ }^{6,16}$, S. Mender ${ }^{7}$, S. Mićanović 22 , D. Miceli ${ }^{3}$, T. Miener ${ }^{9}$, M. Minev $^{33}$, J. M. Miranda ${ }^{12}$, R. Mirzoyan ${ }^{16}$, E. Molina ${ }^{20}$, A. Moralejo ${ }^{17}$, D. Morcuende ${ }^{9}$, V. Moreno ${ }^{25}$, E. Moretti ${ }^{17}$, P. Munar-Adrover ${ }^{25}$, V. Neustroev ${ }^{34}$, C. Nigro ${ }^{17}$, K. Nilsson ${ }^{24}$, D. Ninci ${ }^{17}$, K. Nishijima ${ }^{31}$, K. Noda ${ }^{6}$, S. Nozaki ${ }^{30}$, Y. Ohtani ${ }^{6}$, T. Oka ${ }^{30}$, J. Otero-Santos ${ }^{1,2}$, M. Palatiello ${ }^{3}$, D. Paneque ${ }^{16}$, R. Paoletti ${ }^{12}$, J. M. Paredes ${ }^{20}$, L. Pavletic ${ }^{22}$, P. Peñil ${ }^{9}$, C. Perennes ${ }^{18}$, M. Persic ${ }^{3,39}$, P. G. Prada Moroni ${ }^{19}$, E. Prandinii ${ }^{18}$, C. Priyadarshi ${ }^{17}$, I. Puljak ${ }^{27}$, W. Rhode ${ }^{7}$, M. Ribó ${ }^{20}$, J. Rico ${ }^{17}$, C. Righi ${ }^{4}$, A. Rugliancich ${ }^{19}$, L. Saha ${ }^{9}$, N. Sahakyan ${ }^{26}$, T. Saito ${ }^{6}$, S. Sakurai ${ }^{6}$, K. Satalecka ${ }^{13}$, F. G. Saturni ${ }^{4}$, B. Schleicher ${ }^{23}$, K. Schmidt ${ }^{7}$, T. Schweizer ${ }^{16, \star}$, J. Sitarek ${ }^{11}$, I. Śnidarić ${ }^{35}$, D. Sobczynska ${ }^{11}$, A. Spolon ${ }^{18}$, A. Stamerra ${ }^{4}$, D. Strom ${ }^{16}$,

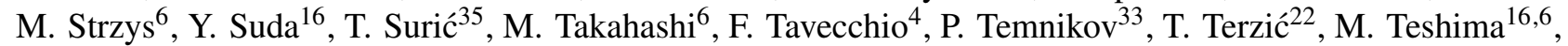
N. Torres-Albà ${ }^{20}$, L. Tosti ${ }^{15}$, S. Truzzi ${ }^{12}$, A. Tutone ${ }^{4}$, J. van Scherpenberg ${ }^{16}$, G. Vanzo $^{1,2}$, M. Vazquez Acosta ${ }^{1,2}$, S. Ventura ${ }^{12}$, V. Verguilov ${ }^{33}$, C. F. Vigorito ${ }^{15}$, V. Vitale ${ }^{15}$, I. Vovk $^{6}$, M. Will ${ }^{16}$, D. Zaric ${ }^{27}$, K. Hirotani ${ }^{40, \star}$, and P. M. Saz Parkinson ${ }^{41,42}$

(Affiliations can be found after the references)

Received 9 August 2020 / Accepted 18 September 2020

\section{ABSTRACT}

We report the detection of pulsed gamma-ray emission from the Geminga pulsar (PSR J0633+1746) between $15 \mathrm{GeV}$ and $75 \mathrm{GeV}$. This is the first time a middle-aged pulsar has been detected up to these energies. Observations were carried out with the MAGIC telescopes between 2017 and 2019 using the low-energy threshold Sum-Trigger-II system. After quality selection cuts, $\sim 80 \mathrm{~h}$ of observational data were used for this analysis. To compare with the emission at lower energies below the sensitivity range of MAGIC, 11 years of Fermi-LAT data above $100 \mathrm{MeV}$ were also analysed. From the two pulses per rotation seen by Fermi-LAT, only the second one, $P 2$, is detected in the MAGIC energy range, with a significance of $6.3 \sigma$. The spectrum measured by MAGIC is well-represented by a simple power law of spectral index $\Gamma=5.62 \pm 0.54$, which smoothly extends the Fermi-LAT spectrum. A joint fit to MAGIC and Fermi-LAT data rules out the existence of a sub-exponential cut-off in the combined energy range at the 3.6 $\sigma$ significance level. The power-law tail emission detected by MAGIC is interpreted as the transition from curvature radiation to Inverse Compton Scattering of particles accelerated in the northern outer gap.

Key words. gamma rays: stars - pulsars: general - pulsars: individual: PSR J0633+1746 - pulsars: individual: Geminga

^ Corresponding authors; e-mail: contact.magic@mpp.mpg.de 


\section{Introduction}

Geminga (PSR J0633+1746) is an archetype of the radioquiet gamma-ray pulsar population (Bignami \& Caraveo 1996; Caraveo 2014). First detected by SAS-2 and COS-B (Fichtel et al. 1975; Hermsen et al. 1977; Bignami \& Caraveo 1992) as a bright gamma-ray source with no counterpart at any other wavelength and subsequently associated with an X-ray source (Bignami et al. 1983), it was ultimately identified as a pulsar by ROSAT and EGRET (Halpern \& Holt 1992; Bertsch et al. 1992). It has a period of $P \simeq 237 \mathrm{~ms}$ and a characteristic age of $\sim 300 \mathrm{ky}$. Two independent measurements of the distance reported $157_{-34}^{+59} \mathrm{pc}$ (Caraveo et al. 1996) and $250_{-62}^{+120} \mathrm{pc}$ (Faherty et al. 2007), respectively. This makes Geminga one of the closest known pulsars.

The Fermi-LAT detector measured the pulsed gamma-ray spectrum of Geminga using one year of data and found that it can be described by a power law with an exponential cutoff at $2.46 \pm 0.04 \mathrm{GeV}$ (Abdo et al. 2010). The increase of Fermi-LAT statistics in the following years favoured a softer sub-exponential cut-off (Abdo et al. 2013; Ahnen et al. 2016). Subsequent ground-based observations by the Imaging Atmospheric Cherenkov Telescopes (IACT) VERITAS (Aliu et al. 2015) and MAGIC (Ahnen et al. 2016) could not detect any significant emission above $100 \mathrm{GeV}$ and $50 \mathrm{GeV}$, respectively. A $\sim 2$ deg steady halo around Geminga was first detected by the MILAGRO experiment (Abdo et al. 2009), and later reported by the HAWC (Abeysekara et al. 2017) and Fermi-LAT (Manconi et al. 2019) collaborations at energies above $5 \mathrm{TeV}$ and $8 \mathrm{GeV}$, respectively.

In this paper, we report the detection of pulsed gamma-ray emission from the Geminga pulsar by the MAGIC telescopes. This makes Geminga the first middle-aged pulsar detected by IACTs and the third pulsar detected by these type of telescopes after the Crab (Aliu et al. 2008) and Vela (Abdalla et al. 2018). This detection had become possible thanks to the use of the new low-energy trigger system, dubbed Sum-Trigger-II (Dazzi et al., in prep.; García et al. 2014), designed to improve the performance of the telescopes in the sub- $100 \mathrm{GeV}$ energy range.

In Sect. 2 we present the MAGIC observations and the technical innovations that were imperative for this detection. The analysis of Fermi-LAT data is described in Sect. 3. The resulting MAGIC and Fermi-LAT light curves and spectra are presented in Sect. 4 and discussed in Sect. 5. Finally, we compare our observations with the predictions of the pulsar outer gap model (Cheng et al. 1986; Romani \& Yadigaroglu 1995) applied to Geminga in Sect. 6.

\section{MAGIC observations and data analysis}

The MAGIC telescopes are two imaging atmospheric Cherenkov telescopes (IACTs) located on the Canary Island of La Palma (Spain) (Aleksić et al. 2016a). Observations of Geminga with the MAGIC Sum-Trigger-II system began in January 2017 and lasted until March 2019. Aiming for the lowest possible energy threshold, the observation zenith angle of the source was limited to below $25 \mathrm{deg}$. Data taken in the period between December 2017 and March 2018 were affected by non-optimal weather conditions and were discarded. After this selection, a total of $80 \mathrm{~h}$ of good quality data were available. The observations were made in the so-called 'wobble mode' (Fomin et al. 1994), in which the telescopes were pointed at sky positions around Geminga with an offset of $0.4 \mathrm{deg}$. Together with each event image, we recorded the event arrival time with a GPS disciplined
Rubidium oscillator, which provides an absolute time stamp precision of $200 \mathrm{~ns}$.

The detection of the Geminga pulsar with MAGIC was possible thanks to the implementation of the Sum-Trigger-II system. The standard MAGIC trigger requires that the signals of three neighbouring camera pixels exceed a preset threshold of $\sim 4.0$ photo electrons (phe.). In the Sum-Trigger-II, the pixels are grouped into hexagonal-shape cells of 19 pixels each. The analogue sum of all pixel signals within any given cell is compared against a discriminator threshold of $\sim 18$ phe. Integrating the signal from a large area leads to a better signal-to-noise ratio $(\mathrm{S} / \mathrm{N})$ for very low energy showers. To counteract the effect of noise after-pulses, which are typical for photo-multiplier tubes, the individual pixel amplitudes are clipped when exceeding 8.5 phe. The trigger geometry, thresholds, and clipping values were optimised by Monte Carlo (MC) simulations with the aim of minimizing the trigger threshold (Dazzi et al., in prep.). Compared to the standard trigger, the trigger energy threshold of the SumTrigger-II is about 50\% lower. For a spectral index of -5 , which is similar to that of Geminga as reported here, the peak of the gamma energy distribution (threshold) is approximately $15 \mathrm{GeV}$.

The MAGIC data were processed with the Magic Standard Analysis Software (MARS, Zanin et al. 2013). To improve the analysis performance close to the Sum-Trigger-II energy threshold, we developed a new algorithm in which the calibration and the image cleaning are performed in an iterative procedure. The rest of the higher level analysis followed the standard pipeline described in Aleksić et al. (2016b). This comprises the reconstruction of the energy and direction of the incoming gamma rays and the suppression of the hadronic background. Boosted decision trees and look-up tables were built for these purposes, using gamma-ray simulated shower events following the trajectory of Geminga in the sky and background events from dedicated observations.

For the timing analysis, the pulsar rotational phases of the events were computed using the Tempo 2 package (Hobbs et al. 2006). An ephemeris for Geminga covering the MAGIC observations was obtained from the analysis of Fermi-LAT data (Kerr et al. 2015).

\section{Fermi-LAT data and analysis}

To characterise the Geminga emission at energies lower than those accessible to MAGIC, we analysed 10.6 years (from MJD 54682 to 58569 ) of public Fermi-LAT data across the energy range from $100 \mathrm{MeV}$ to $2 \mathrm{TeV}$. We processed this data set using the P8R2_SOURCE_V6 instrument response functions and the Fermi Science Tools version v11r5p3. Events were selected within a circular region of interest (ROI) of $15^{\circ}$ centred at the pulsar position $\left(\mathrm{RA}=06^{\mathrm{h}} 33^{\mathrm{m}} 54.29^{\mathrm{s}}\right.$, Dec $\left.=17^{\circ} 46^{\prime} 14^{\prime \prime} .88\right)$. We selected 'Source' class events that were recorded only when the telescope was in nominal science mode. The pulsar rotational phase and barycentric corrections of the events were computed with Tempo2, using the same ephemeris as for the MAGIC data analysis. The pulsar light curve was produced applying an additional energy dependent angular cut, according to the approximation of the Fermi-LAT Pass8 point spread function for a $68 \%$ confinement radius (Acero et al. 2015).

For the spectral reconstruction, a binned likelihood analysis was performed making use of the pyLikelihood python module of the Fermi Science Tools. Each of the two emission peaks of the Geminga light curve, $P 1$ and $P 2$, were analysed separately. We started the likelihood fits by including all sources in the ROI from the third Fermi Source Catalogue (Acero et al. 2015) in the 
(a) Fermi-LAT > $5 \mathrm{GeV}$

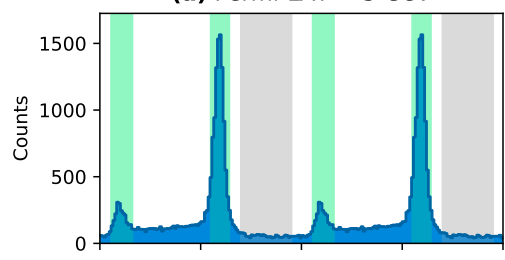

(b) Fermi-LAT > $15 \mathrm{GeV}$

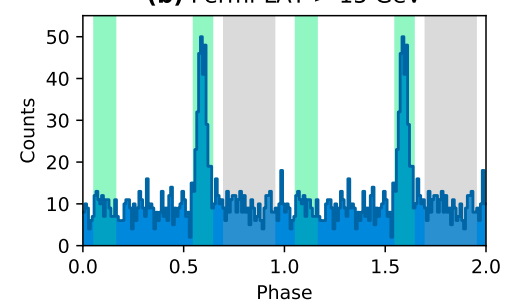

(c) MAGIC $>15 \mathrm{GeV}$

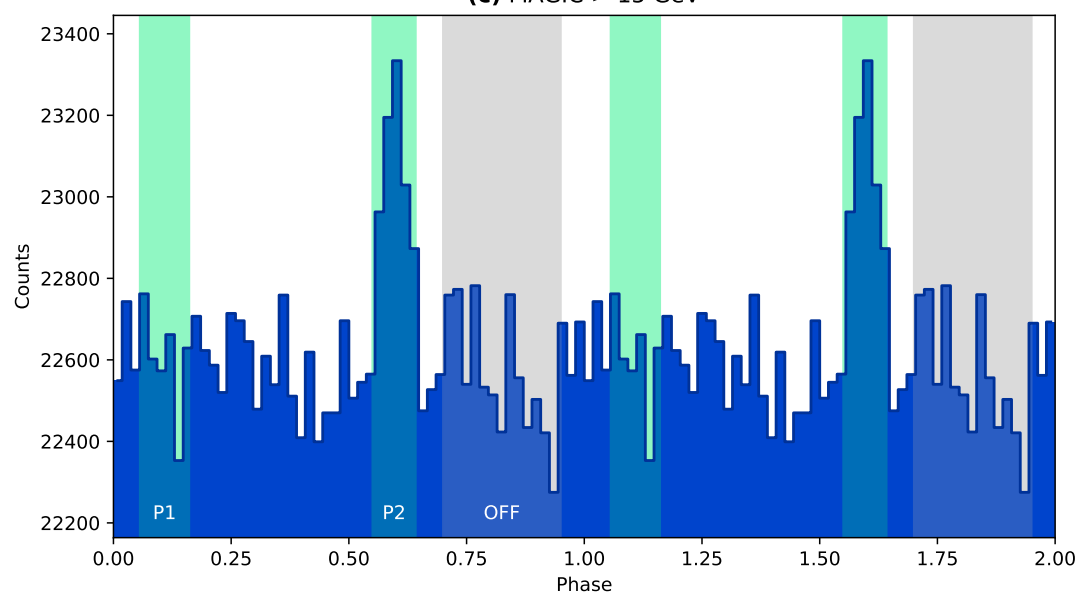

Fig. 1. Geminga light curves measured by Fermi-LAT (panels $a$ and $b$ ), and by MAGIC (panel $c$ ). For clarity, two rotation cycles are shown. The green-shaded regions highlight the phase intervals corresponding to the $P 1$ and $P 2$ emission, obtained from the fits to Fermi-LAT data above 5 GeV and $15 \mathrm{GeV}$, respectively. The phase region from which the background is estimated is shown by the grey band. These signal regions were later applied to the analysis of MAGIC data. $P 2$ is detected with MAGIC at a significance level of $6.25 \sigma$. No significant signal is detected from $P 1$ in the MAGIC energy range.

spectral-spatial model. The spectral parameters for sources with a significance higher than $5 \sigma$ and located within $5 \mathrm{deg}$ of the centre of the ROI were left free. Also, we let the normalisation factor of the isotropic and Galactic background models free. For the rest of the sources, all parameters were fixed to their catalogue values. In a second step, all sources with $T S<4$ were removed from the model. For the calculation of the spectral points, we repeated the procedure in each energy bin using a power law with the normalisation factor free and the spectral index fixed to 2 .

\section{Results}

\subsection{Light curves}

The light curves shown in Fig. 1 are produced by phase folding Fermi-LAT photons and MAGIC events using the same pulsar ephemeris. The two well-known Geminga emission peaks, $P 1$ and $P 2$, are clearly visible above $5 \mathrm{GeV}$ in Fermi-LAT data. At higher energies, only $P 2$ is detected by Fermi-LAT, which is in agreement with the high-energy light curves shown in Ackermann et al. (2013). To characterise each peak at energies as close as possible to the MAGIC energy range, we fit them to symmetric Gaussian functions, using Fermi-LAT events above $5 \mathrm{GeV}$ for $P 1$ and above $15 \mathrm{GeV}$ for $P 2$. The corresponding light curves are shown in Fig. 1, panels a and $b$. The phase signal regions for the analysis of MAGIC data are then defined as the $\pm 2 \sigma$ intervals around the fitted peak positions. For estimating the background, we considered the off-pulse region between $P 2$ and $P 1$, where no emission is expected from the pulsar, starting $6 \sigma$ away from each peak's centre. Table 1 summarises the signal and background regions used for the MAGIC analysis.

The MAGIC light curve for events with reconstructed energies above $15 \mathrm{GeV}$ is shown in Fig. 1, panel c. It was obtained after applying energy-dependent gamma and hadron separation cuts, trained on MC simulated gamma-ray showers. The number of excess events for each emission peak and the corresponding significances were computed using Eq. (17) in Li \& Ma (1983) and they are tabulated in Table 1. Emission from $P 2$ is detected with MAGIC at a significance level of $6.25 \sigma$, corresponding to 2457 excess events over a scaled background of 112018 events.
Table 1. Definition of the signal $(P 1, P 2)$ and background (OFF) regions derived from the analysis of the Fermi-LAT light curves shown in Fig. 1.

\begin{tabular}{rrrr}
\hline \hline \multicolumn{2}{c}{ Phase region } & Excess & $\sigma$ \\
\hline$P 1$ & $(0.056-0.161)$ & 116.7 & 0.27 \\
$P 2$ & $(0.550-0.642)$ & 2457.3 & 6.25 \\
OFF & $(0.700-0.950)$ & & \\
\hline
\end{tabular}

Notes. The last two columns refer to the number of excess events and the significance $\sigma$ (following the Li\&Ma definition) obtained in the analysis of MAGIC data.

A region-independent signal test with the $H$-test and $Z_{10}^{2}$-test (de Jager \& Büsching 2010) results in $4.8 \sigma$ and $5.2 \sigma$ significances, respectively. The analysis of MAGIC events in the phase region of $P 1$ does not reveal any significant signal in this energy range.

\subsection{Energy spectrum}

Figure 2 shows the Spectral Energy Distribution (SED) of Geminga for $P 2$, obtained after the analysis of Fermi-LAT (open circles) and MAGIC data (filled circles). The spill-over effect due to the soft spectral index has been carefully taken into account by unfolding the MAGIC energy spectrum using the Tikhonov regularisation method (Albert et al. 2007), and cross-checked with a forward-folding procedure. The resulting MAGIC unfolded spectral points (filled circles in Figs. 2) are reported in Table 2 . The dashed blue line shows the forwardfolding power-law fit, $F_{0}\left(E / E_{0}\right)^{-\Gamma}$, performed on the distribution of MAGIC excess events. The blue butterfly represent the $1 \sigma$ statistical uncertainty confidence interval of the fit. The spectrum measured by MAGIC in the energy range $15-75 \mathrm{GeV}$ is well-represented by the power law, with an associated $\chi^{2}=$ 15.27 with 15 degrees of freedom. The obtained spectrum is in agreement with the upper limits previously reported by MAGIC (Ahnen et al. 2016). The resulting fit parameters are reported in Table 3. The spectral index $\Gamma=5.62 \pm 0.54$ (statistical errors only) is the softest ever measured by MAGIC from any source. 


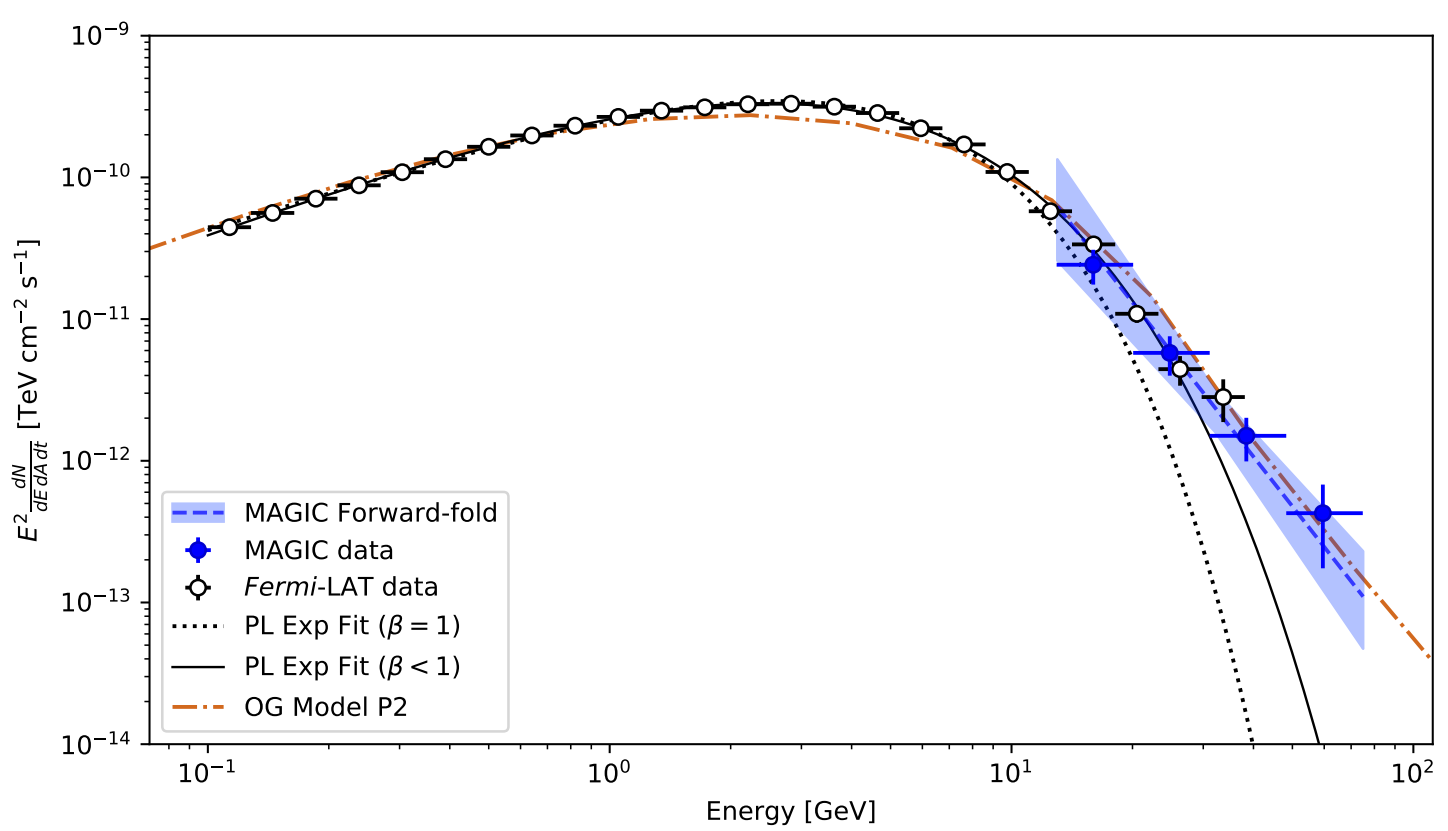

Fig. 2. SED of the second emission peak, $P 2$, of the Geminga pulsar measured by the MAGIC telescopes (filled circles) and the Fermi-LAT (open circles). The MAGIC spectral points were calculated by unfolding the reconstructed energy spectrum by means of the Tikhonov regularisation method. Dashed blue line shows the forward folding fit to the MAGIC data assuming a power law and the blue-shaded area represents the $1 \sigma$ confidence interval around the power-law fit. Dotted and continuous black lines represent the combined fit to MAGIC and Fermi-LAT data to a power law with an exponential or sub-exponential cutoff, respectively. Dotted-dashed orange line shows the $P 2$ spectrum predicted in the stationary outer gap model applied to Geminga for a magnetic dipole moment $\mu=1.4 \mu_{\mathrm{d}}$, an angle between the magnetic and the rotational axis of the star, $\alpha=30^{\circ}$, and an observer's viewing angle, $\zeta=95^{\circ}$.

Table 2. MAGIC SED points.

\begin{tabular}{cccc}
\hline \hline$E_{\text {low }}$ & $E$ & $E_{h i}$ & $E^{2} \mathrm{~d} N /(\mathrm{d} E \mathrm{~d} A \mathrm{~d} t)$ \\
\hline 12.9 & 16.0 & 20.1 & $(2.42 \pm 0.67) \times 10^{-11}$ \\
20.1 & 24.8 & 31.1 & $(5.77 \pm 1.79) \times 10^{-12}$ \\
31.1 & 38.4 & 48.2 & $(1.50 \pm 0.51) \times 10^{-12}$ \\
48.2 & 59.5 & 74.8 & $(4.30 \pm 2.50) \times 10^{-13}$ \\
\hline
\end{tabular}

Notes. The energy bin edges $\left(E_{\text {low }}, E_{h i}\right)$, as well as their centre position, $E$, are in units of $\mathrm{GeV}$. SED values are in $\mathrm{TeV} \mathrm{cm} \mathrm{cm}^{-2} \mathrm{~s}^{-1}$.

\section{Discussion}

We performed a joint fit of MAGIC and Fermi-LAT spectral points in the combined energy range, from $100 \mathrm{MeV}$ to $75 \mathrm{GeV}$, by using a power law with an exponential cut-off function:

$F(E)=F_{0}\left(E / E_{0}\right)^{-\Gamma} \exp \left(-\left(E / E_{\mathrm{c}}\right)^{\beta}\right)$,

where $E_{0}$ is the energy scale, $\Gamma$ the spectral index, $E_{\mathrm{c}}$ the cutoff energy, and $\beta$ the cut-off strength. We considered two different cases: a pure exponential cut-off, $\beta=1$, and the general case in which the $\beta$ parameter is set free. The parameters resulting from the fits are given in Table 3. The best fit is found for $\beta=0.738 \pm 0.013$. In a likelihood-ratio test versus the free exponential cut-off model, the pure exponential case can be rejected with TS $=-2 \Delta \log \mathcal{L}=336.6$, which, according to a chi-square distribution with one degree of freedom, corresponds to a significance of $18.3 \sigma$. Also, the sub-exponential cut-off is disfavoured by the data at the level of $3.6 \sigma$, according to a goodness-of-fit chi-square test.

In order to assess whether there is any preference for curvature in the high energy tail of the spectrum, we fitted MAGIC and Fermi-LAT spectral points above $10 \mathrm{GeV}$ to a log-parabola model, $F_{0}\left(E / E_{0}\right)^{-\Gamma-\beta \log \left(E / E_{0}\right)}$, obtaining a best-fit value for the curvature index of $\beta=0.99_{-0.84}^{+0.67}$. We performed a likelihoodratio test between this model and a power law. This results in $\mathrm{TS}=1.7$ with 1 degree of freedom, corresponding to $1.3 \sigma$ against the power-law model. This shows that the log-parabola model is not significantly preferred over the power-law one. The power-law index derived from the joint fit, $\Gamma=5.18 \pm 0.15$, is compatible with the one obtained with MAGIC data alone.

The effect of systematic uncertainties on the MAGIC spectral reconstruction has been studied. A 5\% change in the estimated energy of the events has little effect in the reconstructed spectral index (below 1\%), but makes the MAGIC fluxes fluctuate by up to $20 \%$. This results from the combined effect of the softness of the Geminga spectrum and the steeply falling effective collection area close to the MAGIC energy threshold. The joint fit with Fermi-LAT data in the overlapping energy range helps to constrain these uncertainties. To test this we introduced a MAGIC flux scale factor, $s$, as a nuisance parameter in the fits. Maximum-likelihood values of the scale parameter are always found to be compatible with unity within the uncertainties, with $s=0.88 \pm 0.11$ and $s=0.98 \pm 0.14$ for the sub-exponential and the log-parabolic fits, respectively. Likelihood-ratio tests versus a model with no scaling provide, in both cases, TS $<1.0$. We conclude that the energy calibration of MAGIC with respect to Fermi-LAT is accurate.

\section{Modelling the high-energy emission}

Two main radiation processes are considered to be responsible for the gamma-ray emission detected in pulsars: synchrocurvature radiation or Inverse Compton Scattering (ICS), or a combination of both. The first can explain the exponential 
Table 3. Results from the spectral fits performed to the MAGIC data alone (first row) to a power law function (PL), and from the joint fits to Fermi-LAT and MAGIC data (abbreviated as F \& M) to power laws with exponential (PL Exp) or sub-exponential (PL Subexp) cut-offs, and to a log-parabola model (LP).

\begin{tabular}{cccccccc}
\hline \hline Data & Function & $F_{0}$ & $E_{0}$ & $\Gamma$ & $E_{c}$ & $\beta$ & $-2 \log \mathcal{L}$ \\
\hline MAGIC & PL & $(2.28 \pm 0.74) \times 10^{-9}$ & 32.15 & $5.62 \pm 0.54$ & - & - & - \\
F \& M & PL Exp & $(0.357 \pm 0.002) \times 10^{-3}$ & 1.00 & $1.089 \pm 0.003$ & $2.88 \pm 0.02$ & 1 & 388.2 \\
F \& M & PL Subexp & $(0.552 \pm 0.019) \times 10^{-3}$ & 1.00 & $0.910 \pm 0.013$ & $1.44 \pm 0.08$ & $0.738 \pm 0.013$ & 51.6 \\
F $>10 \&$ M & PL & $(0.29 \pm 0.04) \times 10^{-8}$ & 32.15 & $5.18 \pm 0.15$ & - & - & 11.1 \\
F $>10 \&$ M & LP & $(0.21 \pm 0.06) \times 10^{-8}$ & 32.15 & $6.4_{-0.9}^{+1.1}$ & - & $0.99_{-0.84}^{+0.67}$ & 9.4 \\
\hline
\end{tabular}

Notes. For the last two fits only Fermi-LAT spectral points above $10 \mathrm{GeV}$ were used. The normalisation factor $F_{0}$ is given in units of TeV $\mathrm{Cm}^{-1} \mathrm{~cm}^{-1}$. The normalisation energy, $E_{0}$, and the cut-off energy, $E_{\mathrm{c}}$, are given in units of GeV. Also, $\Gamma$ refers to the PL spectral index, and $\beta$ to the cut-off strength, except for the LP case for which $\beta$ represents the curvature of LP. The quoted uncertainties are statistical at a $1 \sigma$ confidence level.

cut-offs at a few $\mathrm{GeV}$ seen in the vast majority of FermiLAT pulsars, while the second process may account for the power-law spectral tail detected in the Crab pulsar up to $\mathrm{TeV}$ energies (Ansoldi et al. 2016).

We compare our observational results with the predictions of the stationary three-dimensional pulsar outer gap (OG) model (Hirotani 2006, 2013). We assume that the magnetic field lines are given by the rotating vacuum dipole solution (Cheng et al. 2000; Aliu et al. 2015), and we solve Gauss's law, the stationary Boltzmann equations for electrons and positrons, and the radiative transfer equation of the emitted photons from IR to veryhigh-energy (VHE) gamma rays. Accordingly, we can obtain the pulse profile and the phase-resolved spectrum of the emitted photons, by setting the following five parameters of the neutron star (NS): the rotational angular frequency, $\Omega_{\mathrm{F}}$; the surface temperature, $T$; the area of the star, $A$; the magnetic dipole moment, $\mu$; and the angle $\alpha$ between the NS magnetic and rotational axes. The NS rotational period, $P$, is an observable and readily gives $\Omega_{\mathrm{F}}=2 \pi / P$. Using the soft X-ray data (Halpern $\&$ Wang 1997), we constrain the NS surface emission with temperature $k T=49.74 \mathrm{eV}$ and area $A=0.5085 \times 4 \pi r_{0}{ }^{2}$, where $4 \pi r_{0}{ }^{2}$ denotes the whole NS surface area measured by a distant observer. The distance to source is assumed to be $250 \mathrm{pc}$ (Faherty et al. 2007). We also include in the seed X-ray spectrum a harder component $(k T \sim 185 \mathrm{eV})$ associated with the heated polar cap region discussed in Caraveo et al. (2004). The value of $\mu$ will not be very different from its dipole value $\mu_{\mathrm{d}}$, which is constrained by $P$ and its temporal derivative, $\dot{P}$, under the assumption of magnetic dipole radiation. Therefore, $\mu / \mu_{\mathrm{d}}$, $\alpha$, and the observer's viewing angle with respect to the rotation axis, $\zeta$, remain as free parameters. We constrain these parameters by comparing the predicted pulse profile and phase-resolved spectrum with the MAGIC and Fermi-LAT observations.

For the Geminga pulsar, unlike in young pulsars like the $\mathrm{Crab}$, ICS is negligible in the outer magnetosphere because the IR photon fields are too weak. As a result, the positrons accelerated outward in the gap, produces negligible VHE fluxes. Nevertheless, gap-accelerated electrons continue propagating towards the star to efficiently up-scatter soft X-ray photons from the NS surface by head-on collisions. Accordingly, if we are observing Geminga nearly perpendicularly to its rotation axis, the inward VHE photons emitted by the electrons would appear in the same rotational phase as the outward HE photons emitted by the positrons.

To explain the MAGIC flux and the double-peaked pulse profile observed at lower energies by Fermi-LAT with a peak separation of $183^{\circ}$ (Abdo et al. 2013), we find that $\mu=1.4 \mu_{\mathrm{d}}, \alpha \sim 30^{\circ}$ and $95^{\circ}<\zeta<100^{\circ}$ are necessary. A similar viewing angle of $\zeta=90^{\circ}$ was also obtained in Pierbattista et al. (2015), by fitting the Fermi-LAT light curve on the basis of the OG model. The dotted-dashed orange line in Fig. 2 shows the predicted Geminga flux from the $P 2$ phase region defined in Table 1 for a viewing angle $\zeta=95^{\circ}$. The OG solutions predicting emission in the MAGIC energy range tend to under predict the Fermi-LAT flux at few $\mathrm{GeV}$. This may indicate the limitation of stationary OG models, suggesting the need for non-stationary particle-in-cell simulations of pulsar magnetospheres ${ }^{1}$. At the viewing angle of $\zeta=95^{\circ}$, below $40 \mathrm{GeV}$, the flux of $P 2$ is dominated by the outward photons emitted in the northern OG via mainly the curvature process. Above $40 \mathrm{GeV}$, the $P 2$ flux is instead dominated by the inward photons emitted via ICS in the same northern OG. The emission from the southern OG is relatively small for $P 2$, whereas for $P 1$, the role of northern and southern OGs are exchanged. At $\zeta=100^{\circ}$ the inward ICS emission dominates the outward one above $100 \mathrm{GeV}$. At $\zeta \geq 105^{\circ}$, the ICS component becomes negligible. It also follows that the present stationary OG model predicts an extension of the ICS pulsed component above the energies reported by MAGIC.

\section{Summary}

In this paper, we present the detection of pulsed gamma-ray emission from the Geminga pulsar with the MAGIC telescopes. The emission coincides in pulse phase with the position of $P 2$ and is detected up to $75 \mathrm{GeV}$. This makes Geminga the first middle-aged pulsar detected up to such energies. The spectrum measured by MAGIC is well-described by a power law of spectral index $\Gamma=5.62 \pm 0.54$. A joint fit to MAGIC and Fermi-LAT data rules out the existence of an exponential cut-off in the combined energy range. A sub-exponential cut-off is also disfavoured at the $3.6 \sigma$ level. According to the outer gap model, the emission detected by MAGIC implies that we are observing Geminga nearly perpendicularly to its rotation axis and that the emission originates in the northern outer gap. The energy range covered by MAGIC would correspond to the transition from curvature radiation by outward accelerated positrons to ICS by electrons accelerated towards the star. The ICS component is predicted to extend above the energies detected by MAGIC. This should be confirmed by future Geminga observations by IACTs, as well as by non-stationary pulsar gap models.

\footnotetext{
1 Particle-in-cell simulations (see e.g. Brambilla et al. 2018 and references therein), are currently limited to much weaker magnetic field strengths than the actual values found in pulsar magnetospheres. Consequently, they cannot be used at present to probe pulsar emission above $\mathrm{GeV}$ energies.
} 
Acknowledgements. The authors would like to thank Matthew Kerr for helping in the production of the ephemeris used in this analysis. We would also like to thank the Instituto de Astrofísica de Canarias for the excellent working conditions at the Observatorio del Roque de los Muchachos in La Palma. The financial support of the German BMBF and MPG; the Italian INFN and INAF; the Swiss National Fund SNF; the ERDF under the Spanish MINECO (FPA2017-87859-P, FPA2017-85668-P, FPA2017-82729C6-2-R, FPA2017-82729-C6-6-R, FPA2017-82729-C6-5-R, AYA2015-71042P, AYA2016-76012-C3-1-P, ESP2017-87055-C2-2-P, FPA2017-90566-REDC) the Indian Department of Atomic Energy; the Japanese ICRR, the University of Tokyo, JSPS, and MEXT; the Bulgarian Ministry of Education and Science, National RI Roadmap Project DO1-268/16.12.2019 and the Academy of Finland grant nr. 320045 is gratefully acknowledged. This work was also supported by the Spanish Centro de Excelencia "Severo Ochoa" SEV-2016-0588 and SEV2015-0548, the Unidad de Excelencia "María de Maeztu" MDM-2014-0369 and the "la Caixa" Foundation (fellowship LCF/BQ/PI18/11630012), by the Croatian Science Foundation (HrZZ) Project IP-2016-06-9782 and the University of Rijeka Project 13.12.1.3.02, by the DFG Collaborative Research Centers SFB823/C4 and SFB876/C3, the Polish National Research Centre grant UMO2016/22/M/ST9/00382, by the Brazilian MCTIC, CNPq and FAPERJ, and at HKU by a GRF grant (Project 17307618) from the Hong Kong Government.

\section{References}

Abdalla, H., Aharonian, F., Ait Benkhali, F., et al. 2018, A\&A, 620, A66 Abdo, A. A., Allen, B. T., Aune, T., et al. 2009, ApJ, 700, L127 Abdo, A. A., Ackermann, M., Ajello, M., et al. 2010, ApJ, 720, 272 Abdo, A. A., Ajello, M., Allafort, A., et al. 2013, ApJS, 208, 17 Abeysekara, A. U., Albert, A., Alfaro, R., et al. 2017, Science, 358, 911 Acero, F., Ackermann, M., Ajello, M., et al. 2015, ApJS, 218, 23

Ackermann, M., Ajello, M., Allafort, A., et al. 2013, ApJS, 209, 34 Ahnen, M. L., Ansoldi, S., Antonelli, L. A., et al. 2016, A\&A, 591, A138 Albert, J., Aliu, E., Anderhub, H., et al. 2007, Nucl. Instrum. Methods Phys. Res. A, 583, 494

Aleksić, J., Ansoldi, S., Antonelli, L. A., et al. 2016a, Astropart. Phys., 72, 61 Aleksić, J., Ansoldi, S., Antonelli, L. A., et al. 2016b, Astropart. Phys., 72, 76 Aliu, E., Anderhub, H., Antonelli, L. A., et al. 2008, Science, 322, 1221 Aliu, E., Archambault, S., Archer, A., et al. 2015, ApJ, 800, 61

Ansoldi, S., Antonelli, L. A., Antoranz, P., et al. 2016, A\&A, 585, A133 Bertsch, D. L., Brazier, K. T. S., Fichtel, C. E., et al. 1992, Nature, 357, 306 Bignami, G. F., \& Caraveo, P. A. 1992, Nature, 357, 287

Bignami, G. F., \& Caraveo, P. A. 1996, ARA\&A, 34, 331

Bignami, G. F., Caraveo, P. A., \& Lamb, R. C. 1983, ApJ, 272, L9

Brambilla, G., Kalapotharakos, C., Timokhin, A. N., Harding, A. K., \& Kazanas, D. 2018, ApJ, 858, 81

Caraveo, P. A. 2014, ARA\&A, 52, 211

Caraveo, P. A., Bignami, G. F., Mignani, R., \& Taff, L. G. 1996, ApJ, 461, L91

Caraveo, P. A., De Luca, A., Mereghetti, S., Pellizzoni, A., \& Bignami, G. F. 2004, Science, 305, 376

Cheng, K. S., Ho, C., \& Ruderman, M. 1986, ApJ, 300, 500

Cheng, K. S., Ruderman, M., \& Zhang, L. 2000, ApJ, 537, 964

de Jager, O. C., \& Büsching, I. 2010, A\&A, 517, L9

Faherty, J., Walter, F. M., \& Anderson, J. 2007, Ap\&SS, 308, 225

Fichtel, C. E., Hartman, R. C., Kniffen, D. A., et al. 1975, ApJ, 198, 163

Fomin, V. P., Stepanian, A. A., Lamb, R. C., et al. 1994, Astropart. Phys., 2, 137

García, J. R., Dazzi, F., Häfner, D., et al. 2014, ArXiv e-prints [arXiv:1404.4219]

Halpern, J. P., \& Holt, S. S. 1992, Nature, 357, 222

Halpern, J. P., \& Wang, F. Y. H. 1997, ApJ, 477, 905

Hermsen, W., Swanenburg, B. N., Bignami, G. F., et al. 1977, Nature, 269, 494

Hirotani, K. 2006, ApJ, 652, 1475

Hirotani, K. 2013, ApJ, 766, 98

Hobbs, G. B., Edwards, R. T., \& Manchester, R. N. 2006, MNRAS, 369, 655

Kerr, M., Ray, P. S., Johnston, S., Shannon, R. M., \& Camilo, F. 2015, ApJ, 814 128

Li, T. P., \& Ma, Y. Q. 1983, ApJ, 272, 317

Manconi, S., Di Mauro, M., \& Donato, F. 2019, Phys. Rev. D, 100, 123015

Pierbattista, M., Harding, A. K., Grenier, I. A., et al. 2015, A\&A, 575, A3

Romani, R. W., \& Yadigaroglu, I. A. 1995, ApJ, 438, 314

Zanin, R., Carmona, E., Sitarek, J., et al. 2013, Proceedings of the 33rd International Cosmic Ray Conference (ICRC2013): Rio de Janeiro, Brazil, July 2-9, 2013, 0773

\footnotetext{
1 Inst. de Astrofísica de Canarias, 38200 La Laguna, Spain

2 Universidad de La Laguna, Dpto. Astrofísica, 38206 La Laguna, Tenerife, Spain
}

3 Università di Udine and INFN Trieste, 33100 Udine, Italy

${ }^{4}$ National Institute for Astrophysics (INAF), 00136 Rome, Italy

5 ETH Zürich, 8093 Zürich, Switzerland

${ }^{6}$ Japanese MAGIC Group: Institute for Cosmic Ray Research (ICRR), The University of Tokyo, Kashiwa 277-8582, Chiba, Japan

7 Technische Universität Dortmund, 44221 Dortmund, Germany

8 Croatian MAGIC Group: University of Zagreb, Faculty of Electrical Engineering and Computing (FER), 10000 Zagreb, Croatia

9 IPARCOS Institute and EMFTEL Department, Universidad Complutense de Madrid, 28040 Madrid, Spain

10 Centro Brasileiro de Pesquisas Físicas (CBPF), 22290-180 URCA Rio de Janeiro (RJ), Brazil

11 University of Lodz, Faculty of Physics and Applied Informatics, Department of Astrophysics, 90-236 Lodz, Poland

12 Università di Siena and INFN Pisa, 53100 Siena, Italy

13 Deutsches Elektronen-Synchrotron (DESY), 15738 Zeuthen, Germany

14 Centro de Investigaciones Energéticas, Medioambientales y Tecnológicas, 28040 Madrid, Spain

15 Istituto Nazionale Fisica Nucleare (INFN), 00044 Frascati (Roma), Italy

16 Max-Planck-Institut für Physik, 80805 München, Germany

17 Institut de Física d'Altes Energies (IFAE), The Barcelona Institute of Science and Technology (BIST), 08193 Bellaterra (Barcelona), Spain

18 Università di Padova and INFN, 35131 Padova, Italy

19 Università di Pisa and INFN Pisa, 56126 Pisa, Italy

20 Universitat de Barcelona, ICCUB, IEEC-UB, 08028 Barcelona, Spain

21 Armenian MAGIC Group: A. Alikhanyan National Science Laboratory, Yerevan, Armenia

22 Croatian MAGIC Group: University of Rijeka, Department of Physics, 51000 Rijeka, Croatia

23 Universität Würzburg, 97074 Würzburg, Germany

${ }^{24}$ Finnish MAGIC Group: Finnish Centre for Astronomy with ESO, University of Turku, 20014 Turku, Finland

25 Departament de Física, and CERES-IEEC, Universitat Autònoma de Barcelona, 08193 Bellaterra, Spain

26 Armenian MAGIC Group: ICRANet-Armenia at NAS RA, Yerevan, Armenia

27 Croatian MAGIC Group: University of Split, Faculty of Electrical Engineering, Mechanical Engineering and Naval Architecture (FESB), 21000 Split, Croatia

28 Croatian MAGIC Group: Josip Juraj Strossmayer University of Osijek, Department of Physics, 31000 Osijek, Croatia

29 Japanese MAGIC Group: RIKEN, Wako, Saitama 351-0198, Japan

30 Japanese MAGIC Group: Department of Physics, Kyoto University, 606-8502 Kyoto, Japan

31 Japanese MAGIC Group: Department of Physics, Tokai University, Hiratsuka 259-1292, Kanagawa, Japan

32 Saha Institute of Nuclear Physics, HBNI, 1/AF Bidhannagar, Salt Lake, Sector-1, Kolkata 700064, India

33 Inst. for Nucl. Research and Nucl. Energy, Bulgarian Academy of Sciences, 1784 Sofia, Bulgaria

34 Finnish MAGIC Group: Astronomy Research Unit, University of Oulu, 90014 Oulu, Finland

35 Croatian MAGIC Group: Ruđer Bošković Institute, 10000 Zagreb, Croatia

36 Now at University of Innsbruck, Innsbruck, Austria

37 Also at Port d'Informació Científica (PIC), 08193 Bellaterra (Barcelona), Spain

38 Also at Dipartimento di Fisica, Università di Trieste, 34127 Trieste, Italy

39 Also at INAF-Trieste and Dept. of Physics \& Astronomy, University of Bologna, Bologna, Italy

40 Academia Sinica, Institute of Astronomy and Astrophysics (ASIAA), PO Box 23-141, Taipei, Taiwan

41 Department of Physics and Laboratory for Space Research, University of Hong Kong, Pokfulam Road, Pok Fu Lam, Hong Kong

42 Also at Santa Cruz Institute for Particle Physics, University of California, Santa Cruz, CA 95064, USA 